Article

\title{
Safety Assessment of Nano-Hydroxyapatite as an Oral Care Ingredient according to the EU Cosmetics Regulation
}

\author{
Joana M. Ramis ${ }^{1,2, * \mathbb{D}}$, Catarina C. Coelho ${ }^{3,4,5,6 \mathbb{D}}$, Alba Córdoba ${ }^{1,7}$, Paulo A. Quadros ${ }^{6} \mathbb{D}$ \\ and Marta Monjo 1,2,*(D) \\ 1 Cell Therapy and Tissue Engineering Group, Research Institute on Health Sciences (IUNICS), \\ University of the Balearic Islands, 07122 Palma, Spain; albacor81@hotmail.com \\ 2 Department of Fundamental Biology and Health Sciences, University of the Balearic Islands, \\ 07122 Palma, Spain \\ 3 i3S-Instituto de Investigação e Inovação em Saúde, Universidade do Porto, 4200-135 Porto, Portugal; \\ catarina.coelho@fluidinova.pt \\ 4 INEB-Instituto de Engenharia Biomédica, Universidade do Porto, 4200-135 Porto, Portugal \\ 5 Faculdade de Engenharia da Universidade do Porto, Universidade do Porto, 4200-465 Porto, Portugal \\ 6 FLUIDINOVA, S.A., 4470-605 Maia, Portugal; paulo.quadros@fluidinova.com \\ 7 Balearic Islands Health Research Institute (IdISBa), 07010 Palma, Spain \\ * Correspondence: joana.ramis@uib.es (J.M.R.); marta.monjo@uib.es (M.M.); Tel.: +34-971-259-607
}

Received: 26 July 2018; Accepted: 6 September 2018; Published: 11 September 2018

\begin{abstract}
Hydroxyapatite nanoparticles (HAP-NP) are incorporated in oral care products such as toothpastes and mouthwashes to treat dental sensitivity or to promote enamel remineralisation. Despite the good performance of HAP-NP in this application, it is important to ensure its safety for consumers. For that reason, the Scientific Committee on Consumer Safety (SCCS) evaluated the safety of HAP-NP as an oral care ingredient, but the issued opinion was not completely conclusive and the SCCS recommended that additional tests should be performed. Here, we used a commercially available human gingival epithelium (HGE) as a non-animal alternative and MTT cell viability, LDH activity, and IL-1alpha production were evaluated after 3.1\% HAP-NP treatment for $10 \mathrm{~min}$, $1 \mathrm{~h}$, and $3 \mathrm{~h}$. Moreover, the absorption of HAP-NP in the gingival tissue was assessed by transmission electron microscopy (TEM) analysis. Finally, the dissolution behaviour of HAP-NP in simulated gastric fluid was also investigated. No deleterious effect was observed for HGE tissues incubated with HAP-NP for all time-points and parameters evaluated. Moreover, a complete dissolution of $3.1 \%$ HAP-NP in simulated gastric fluid was observed after $7.5 \mathrm{~min}$ at $37^{\circ} \mathrm{C}$. In conclusion, our results evidence the safety of HAP-NP for oral care products with the use of an in vitro replacement alternative for human gingival epithelium and a simulated gastric fluid assay.
\end{abstract}

Keywords: hydroxyapatite nanoparticles; human gingival epithelium; oral care; EU cosmetics regulation; safety evaluation; in vitro biocompatibility; dissolution in simulated gastric fluid

\section{Introduction}

Hydroxyapatite is an extensively used calcium phosphate for bone regenerative applications in orthopaedics and dentistry [1]. Hydroxyapatite nanoparticles (HAP-NP) are very similar to natural hydroxyapatite in terms of size and crystal structure, which contributes to their use in oral care treatments [2]. In recent years, HAP-NP have been incorporated in oral care products such as toothpastes and mouthwashes with the aim of treating dental sensitivity by occluding the dentinal tubules that are opened to the dentin surface and connected to the pulp; or with the purpose of 
promoting enamel remineralisation by replacing calcium and phosphate ions to areas from which minerals have dissolved, restoring its integrity and gloss [2-7].

Safety of cosmetic raw materials has to be demonstrated in order to be approved, yet the Cosmetics Directive establishes the prohibition to test finished cosmetic products and cosmetic ingredients on animals [8]. Having this in mind and in order to assess the safety of a toothpaste ingredient, it should be taken into account that the toothpaste will be in contact with gingival tissue only for a short period of time ( 2 min per 3 times a day); However, this period could be potentially enough for a mouth cellular uptake. In addition, the ingredient could be swallowed and absorbed through the gastrointestinal track and potentially become systemically available [9].

To study the biocompatibility of the HAP-NP in human oral gingiva, in vitro 3D histotypic cultures that closely resemble the histology of the native tissue can be used as an animal alternative for cosmetic ingredient testing $[10,11]$. In fact, the use of in vitro replacement alternatives for skin corrosion and skin irritation testing using commercialised human skin models has been validated by the Scientific Committee on Consumer Safety (SCCS) [12].

In the last years, SCCS has drawn the attention to possible emerging public health problems like the incorporation of nanomaterials in cosmetic products. In this situation, the SCCS group of scientific experts investigate and issue official opinions that are determinant to define the EU legislation [13]. Particularly for hydroxyapatite nanoparticles, a safety assessment has been done by the SCCS in 2016, containing an exhaustive bibliographic analysis regarding the toxicological effects of this nanomaterial as a cosmetic ingredient. However, the SCCS opinion about hydroxyapatite nanoparticles was not completely conclusive, since the toxicological effect of nanoparticles may depend on physical aspects such as size and morphology. For that reason, SCCS demands that the toxicological assessment must be performed for each type of nanomaterial individually in order to prove its safety for consumers [14]. Therefore, the aim of the present study was to assess biocompatibility of a specific HAP-NP to clarify the safety of this ingredient for oral care applications. For that purpose, a commercially available human gingival epithelium reconstructed from primary cells [15] was used, and parameters such as MTT cell viability, LDH activity, and IL-1alpha production were evaluated. Moreover, in order to study the possibility that HAP-NP could become systemically available through absorption in the gingival tissue, transmission electron microscopy (TEM) images of the tissue after different exposure times were evaluated. Finally, the dissolution behaviour in simulated gastric fluid was investigated, so as to assess the possibility of absorption through the intestinal cells after accidental ingestion.

\section{Materials and Methods}

\subsection{Materials and Physico-Chemical Characterization}

\subsubsection{Hydroxyapatite Nanoparticles}

The HAP-NP (CAS 12167-74-7, also referred as 1306-06-5) used in this study were obtained from a commercial formulation (nanoXIM ${ }^{\circledR}$ ) supplied by FLUIDINOVA, S.A. This formulation consisted in a $15.5 \%$ hydroxyapatite nanoparticles suspension in water. Depending on the test performed, the nanoparticles were processed accordingly.

\subsubsection{Transmission Electron Microscopy}

HAP-NP size and morphology was evaluated using transmission electron microscopy (TEM). To perform the analysis, nanoparticles were diluted in ethanol and placed in an ultrasonic bath for $30 \mathrm{~min}$ to disperse the particles. Afterwards, the samples were mounted on copper grids coated with a carbon film and dried inside a desiccator. Finally, the nanoparticles were visualized using a Hitachi HD2700 equipment (Hitachi, Tokyo, Japan). 


\subsubsection{X-ray Diffraction}

HAP-NP phase purity was assessed taking into the international standard "ISO 13779-3: Implants for surgery-Hydroxyapatite-Part 3: Chemical analysis and characterization of crystallinity and phase purity". For that purpose, the HAP-NP suspension was dried overnight at $105{ }^{\circ} \mathrm{C}$ to remove the water and milled to obtain a fine powder. X-ray diffraction (XRD) analysis was performed using a PANalytical Empyrean diffractometer (Malvern Panalytical, Royston, United Kingdom) with the following set up: Step scan time of $198.6450 \mathrm{~s}$, step size $2 \theta$ of $0.0130^{\circ}$, and a $2 \theta$ range between 27 and $39^{\circ}$.

\subsubsection{Surface Area}

The specific surface area of the HAP-NP was analysed using Brunauer-Emmett-Teller (BET) method. For that purpose, the nanoparticles suspension was placed overnight in a drying oven at $105{ }^{\circ} \mathrm{C}$ to remove the water and the solid content was milled to obtain a fine powder. Surface area was determined using a Quantachrome NOVA 2000e equipment (Quantachrome Instruments, Boynton Beach, FL, USA) with sample degasification performed at $300{ }^{\circ} \mathrm{C}$ during $2 \mathrm{~h}$.

\subsection{In Vitro Biocompatibility}

\subsubsection{Reconstructed Human Gingival Epithelium}

The SkinEthic ${ }^{\mathrm{TM}}$ reconstructed human gingival epithelium (HGE) tissue model (EpiSkin, Lyon, France) was used as in vitro model to perform the biocompatibility and irritation tests. The HGE model is composed of normal human gingival cells cultivated on an inert polycarbonate filter at the air liquid interface in a chemically defined medium. This model is histologically similar to the outer cell layers of the human gum.

Upon arrival, the tissues were processed following the manufacturer protocol. Briefly, the tissues were removed from the agarose-nutrient solution in which they are embedded when shipped and were placed in a plate previously filled with the SkinEthic ${ }^{\mathrm{TM}}$ Maintenance Medium (EpiSkin, Lyon, France) at room temperature. The tissues were incubated in a cell incubator at $37^{\circ} \mathrm{C}, 5 \% \mathrm{CO}_{2}$, and saturated humidity for $48 \mathrm{~h}$.

\subsubsection{Treatment with the Hydroxyapatite Nanoparticles}

The maximum incorporation of the HAP-NP aqueous suspension (nanoXIM ${ }^{\circledR}, 15.5 \pm 0.5 \%$ hydroxyapatite nanoparticles aqueous suspension-FLUIDINOVA, S.A., Maia, Portugal) in a toothpaste is $20 \%$. Considering this, it was decided to prepare a $40 \%$ dilution of the HAP-NP aqueous suspension in ultrapure milliQ water (Millipore system, Billerica, MA, USA) and afterwards diluted 1:1 in PBS (phosphate buffered saline), resulting in a final concentration of $20 \%$ dilution of the HAP-NP aqueous suspension, which corresponds to 3.1\% HAP-NP. As negative control, 1:1 milliQ water dilution in PBS was used and as positive control, a 1\% SDS (Sigma-Aldrich, St. Louis, MO, USA) solution was prepared in water and diluted 1:1 in PBS with a final concentration of $0.5 \%$ SDS. Volumes of $40 \mu \mathrm{L}$ of each sample or control were inserted atop of the reconstructed HGE samples and incubated at $37^{\circ} \mathrm{C}$ for the different exposure times (10 $\mathrm{min}, 1 \mathrm{~h}$, and $3 \mathrm{~h}$ ).

\subsubsection{LDH Activity}

Lactate dehydrogenase (LDH) activity in the culture media at the end of the incubation period was used as an index of cell death. LDH activity was determined spectrophotometrically after $30 \mathrm{~min}$ incubation at $25^{\circ} \mathrm{C}$ of $50 \mu \mathrm{L}$ of culture and $50 \mu \mathrm{L}$ of the reaction mixture by measuring the oxidation of $\mathrm{NADH}$ at $490 \mathrm{~nm}$ in the presence of pyruvate, according to the manufacturer's kit instructions (Roche Diagnostics, Mannheim, Germany). Results are presented as percentage of the LDH activity compared to negative control for each time point. 


\subsubsection{MTT Test}

For each of the tested products or controls, at the end of the incubation period, the tissues were rinsed with PBS and placed on $300 \mu \mathrm{L}$ of $0.5 \mathrm{mg} / \mathrm{ml}$ MTT (3-(4,5-dimethylthiazol-2-yl)2,5-diphenyltetrazolium bromide, ThermoFisher Scientific, Waltham MA, USA). After 3 h of incubation at $37^{\circ} \mathrm{C}$ and $5 \% \mathrm{CO}_{2}$, cultures were placed in $2 \mathrm{ml}$ of isopropanol. Extraction was performed overnight at room temperature. Optical density was measured on $200 \mu \mathrm{L}$ of extracts at $570 \mathrm{~nm}$ (reference filter: $690 \mathrm{~nm}$ ). Results are expressed as percentage of viability compared to negative control for each time point.

\subsubsection{IL-1alpha Determination}

At the end of the incubation period, media was collected for the determination of IL-1alpha released to the media. The commercially available Human IL-1 alpha ELISA Kit (ThermoFisher Scientific, Waltham, MA, USA) was run according to supplier instructions. Results are expressed in pg of IL-1alpha per ml of culture media for each group at the different time points.

\subsubsection{Histology}

After incubation period, the tissues were fixed in a balanced $10 \%$ formalin solution and later embedded in paraffin. Four micron vertical sections were stained with hematoxylin/eosin and photographed with a microscope. Hematoxylin/eosin results obtained with the test sample were compared to the positive (damaged tissue) and to the negative (regular tissue) control and scored according to the scale showed in Table 1 [10].

Table 1. Histopathology scoring scale for human gingival epithelium (HGE) tissues.

\begin{tabular}{cl}
\hline Score & \multicolumn{1}{c}{ Description of observations } \\
\hline 0 & $\begin{array}{l}\text { Absence of, or minor epithelium changes } \\
\text { Slight epithelium changes: Slight edema and/or slight cellular changes and/or upper cell layer disintegration } \\
\text { Moderate epithelium changes: Moderate edema and/or moderate cellular alterations and/or presence of } \\
\text { significant number of necrotic cells and/or disintegration of superficial cell layers }\end{array}$ \\
2 & $\begin{array}{l}\text { Marked to severe epithelium changes: Marked edema and/or cellular alterations and or partial tissue } \\
\text { necrosis and/or disintegration of supra basal cell layers } \\
3\end{array}$ \\
4 & Total tissue necrosis and/or tissue disintegration \\
\hline
\end{tabular}

\subsubsection{Observation with TEM}

For each of the tested products or controls, at the end of the incubation period one culture was fixed in a balanced $2.5 \%$ glutaraldehyde solution (Sigma-Aldrich, St. Louis, MO, USA), dehydrated, and later embedded in resin. Vertical sections of $0.3 \mu \mathrm{m}$ were photographed under a Hitachi H600 TEM microscope (Hitachi, Tokyo, Japan).

\subsection{HAP-NP Dissolution in Simulated Gastric Fluid}

\subsubsection{Composition of Simulated Gastric Fluid (SGF)}

SGF was prepared according to the United States Pharmacopeia XXII, containing $0.2 \%(w / v) \mathrm{NaCl}$ (Extrapure, Ph USP grade, Scharlau, Barcelona, Spain) and 0.7\% $(v / v) \mathrm{HCl} 38 \%$ (Molecular Biology tested, Sigma-Aldrich, St. Louis, MO, USA) in ultrapure milliQ water (Millipore system, Billerica, MA, USA). The $\mathrm{pH}$ of the SGF solution was 1.2. The SGF did not contain pepsin since it does not play an active role in the solubilisation of the HAP-NP. The SGF solution was filtered through a $0.2 \mu \mathrm{m}$ pore syringe membrane before the incubation with hydroxyapatite nanoparticles (HAP-NP).

\subsubsection{Calcium Content in the Digestion Media}

According to the SCCS Notes [12], the maximum daily exposure for a mouthwash is $2.16 \mathrm{~g}$ per day and the maximum daily exposure for a toothpaste is $0.138 \mathrm{~g}$ per day. Thus, the stability 
in SGF was evaluated considering the maximum exposure, which corresponds to the mouthwash. Moreover, the maximum incorporation of the HAP-NP aqueous suspension in a mouthwash is $10 \%$ according to the supplier. To significantly increase the safe margin of this test, it was assumed twice that concentration, which is $20 \%$ dilution of HAP-NP aqueous suspension (3.1\% HAP-NP). Therefore, a $2.16 \mathrm{~g}$ solution containing 3.1\% HAP-NP in pure water was added to $250 \mathrm{ml}$ of SGF, following the recommendations of the FDA for the Dissolution Testing of Immediate Release Solid Oral Dosage Forms [16].

Digestions were carried out in a closed flask with a magnetic stirrer at $100 \mathrm{rpm}$ on a heating plate with temperature control at $37^{\circ} \mathrm{C}$, using a Sensoterm II temperature gauge in a thermostatic bath (JP Selecta, Barcelona, Spain). Samples were incubated for 7.5, 15, or 30 min. After incubation, three aliquots of $2 \mathrm{~mL}$ were taken and immediately centrifuged at $20,000 \times \mathrm{g}$ for $30 \mathrm{~min}$ at $25^{\circ} \mathrm{C}$ in an Eppendorf centrifuge 5804R (Eppendorf, Hamburg, Germany). Volumes of $1 \mathrm{~mL}$ of the supernatants were diluted to a final volume of $5 \mathrm{ml}$ in $\mathrm{HNO}_{3} 2 \%(v / v)$.

The $\mathrm{Ca}^{2+}$ concentration in the digestion media was determined by Inductively Coupled Plasma-Atomic Emission Spectroscopy (ICP-AES) (Optima 5300 DV, PerkinElmer, MA, USA). A standard curve was obtained from $\mathrm{Ca}^{2+}$ standard solutions in $\mathrm{HNO}_{3} 2 \%$. Blank samples containing SGF were also analysed and their mean intensity was subtracted from the sample values.

\subsection{Statistics}

All data are presented as mean values \pm SEM. The Kolmogorov-Smirnov test was done to assume parametric or non-parametric distributions for the normality tests. Differences between groups were assessed by Student t-test in case of normal distribution or Mann-Whitney test in case of non-normal distribution. Results were considered statistically significant at $p$-values $<0.05$. SPSS ${ }^{\circledR}$ program for Windows (SPSS Inc, version 17.0, Chicago, IL, USA) was used.

\section{Results}

\subsection{Hydroxyapatite Nanoparticles Physico-Chemical Characterization}

Size and morphology of the hydroxyapatite nanoparticles was investigated using TEM (Figure 1A). As it can be observed, the hydroxyapatite nanoparticles used in this study possess a rod-like morphology and a particle size $<100 \mathrm{~nm}$. Particularly, the HAP-NP morphology show a width between 5-20 nm (typically close to $10 \mathrm{~nm}$ ) and length bellow $50 \mathrm{~nm}$ (typically between 20 to $40 \mathrm{~nm}$ ).

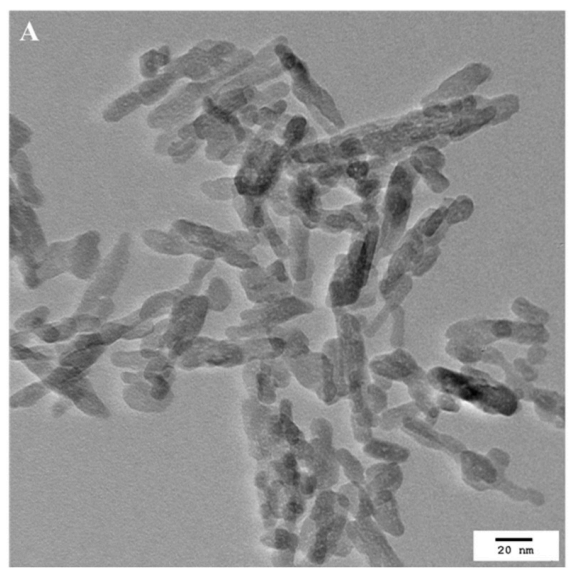

\section{B}

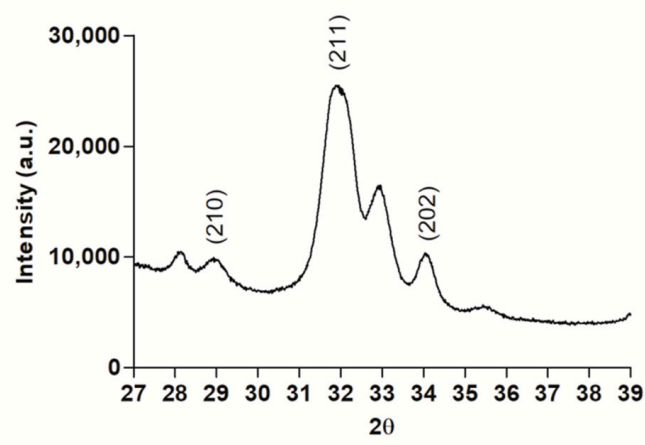

Figure 1. Physicochemical characterization of hydroxyapatite nanoparticles (HAP-NP). (A) transmission electron microscopy (TEM) image of HAP-NP evidencing their particle size and rod-like morphology; (B) X-ray diffraction (XRD) pattern obtained for hydroxyapatite nanoparticles with its characteristic peaks. 
XRD was performed to evaluate the phase composition of the hydroxyapatite nanoparticles. As it can be observed in Figure 1B, the hydroxyapatite was phase pure as the spectrum obtained does not show any unexpected phases. Namely, the peaks positions are in accordance with the ISO standard 13779-3 requirements, as hydroxyapatite is distinguishable by peaks 202, 210, and 211 matched by JCPDS 9-432 file.

Finally, the specific surface area of the hydroxyapatite nanoparticles was evaluated with BET method and the obtained value was $103 \mathrm{~m}^{2} / \mathrm{g}$.

\subsection{Biocompatibility of Hydroxyapatite Nanoparticles}

The LDH assay was used as an indicator of cytotoxicity, as this enzyme leaks out through the plasma membrane of damaged cells. As shown in Figure 2A, 3.1\% HAP-NP did not induce cytotoxicity in HGE tissues at any of the incubation times evaluated. It was also observed that positive control only showed cytotoxicity after $3 \mathrm{~h}$ of incubation.

A

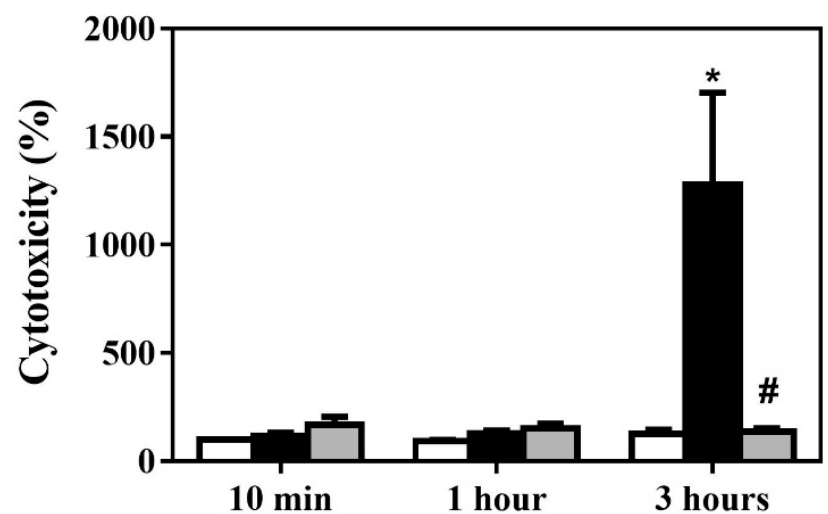

$\square$ Negative control

- Positive control

घ 3.1\% HAP-NP

B

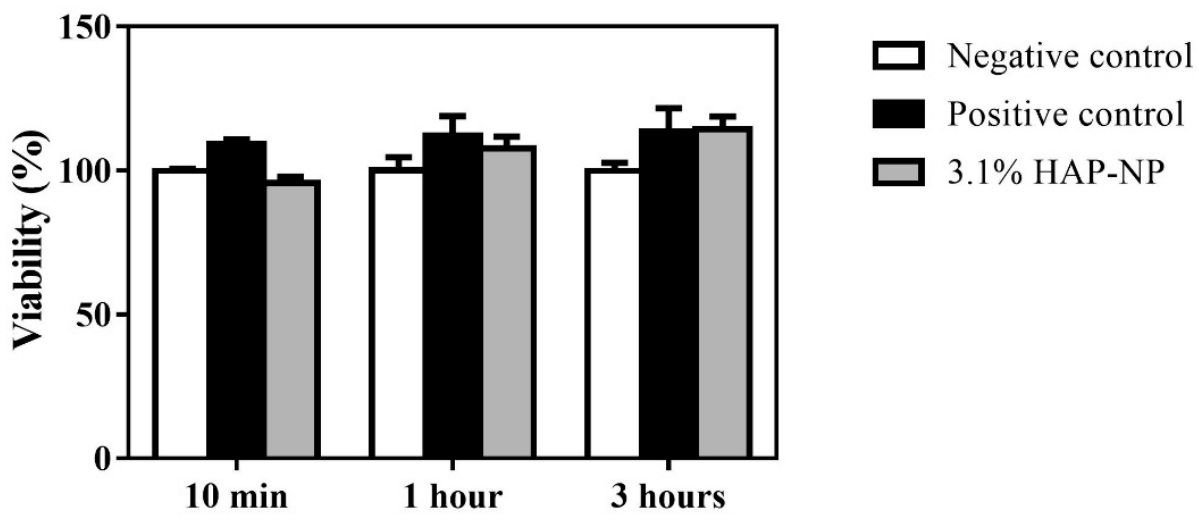

Figure 2. Cont. 


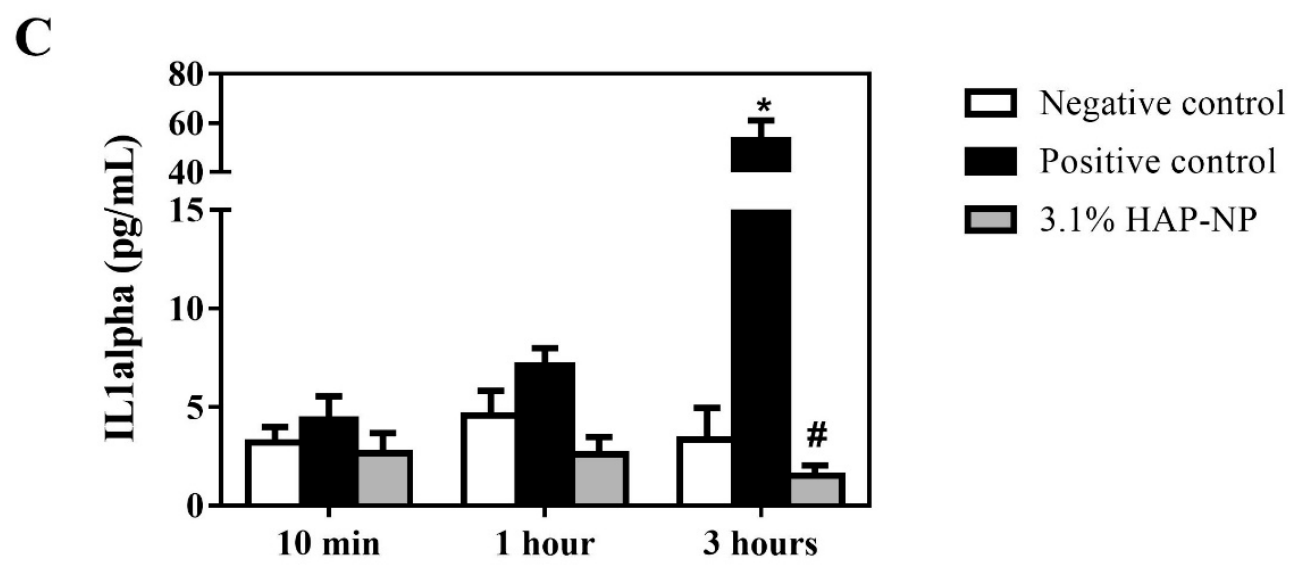

Figure 2. Biocompatibility in HE after different exposure times with the treatments. (A) Lactate dehydrogenase (LDH) activity, an indicator of cytotoxicity, measured in culture media at the end of the different exposure times human gingival epithelium (HGE) with the sample and controls; (B) MTT, an indicator of the number of viable cells, measured in the HGE tissues at the end of the different exposure times with the sample and controls; (C) IL-1alpha, a pro-inflammatory cytokine that is released after cell membrane damage, measured in culture media at the end of the different exposure times of HGE with the sample and controls. Negative control was obtained from culture media treated with milliQ water: PBS (1:1), for LDH and MTT negative control was set at $100 \%$ at each time point. Positive control was obtained from culture media treated with 1\% SDS diluted in PBS (1:1). Test sample was 3.1\% HAP-NP in PBS. Values represent the mean \pm SEM. Mann-Whitney test: ${ }^{*} p<0.05$ versus Negative control; \# $p<0.05$ versus Positive control.

The MTT assay was used as a marker of cell viability, as it measures the MTT reduction by mitochondrial reductase enzymes. As shown in Figure 2B, 3.1\% HAP-NP showed no effect on cell viability at any of the incubation times tested. Similar to the cytotoxicity results, no significant deleterious effect was observed the positive control on the HGE tissue.

The release of IL-1alpha was measured as an indicator of acute irritation in reconstructed human epidermis [17]. As it can be observed in Figure 2C, this test revealed higher values for the positive control that were increased over time. Significant differences comparing the positive control to the rest of groups were observed after $3 \mathrm{~h}$. The concentration of 3.1\% HAP-NP showed similar values to the negative control indicating the absence of acute irritation.

\subsection{Histologycal Results and TEM Observation}

In agreement with the cytotoxicity and IL-1alpha levels results, there was no sign of tissue changes (Figure 3 and Table 2) after incubation with the negative control and the 3.1\% HAP-NP solution. Tissue changes were only observed after treatment with the positive control SDS $0.5 \%$ after 1 and $3 \mathrm{~h}$ of exposure. 


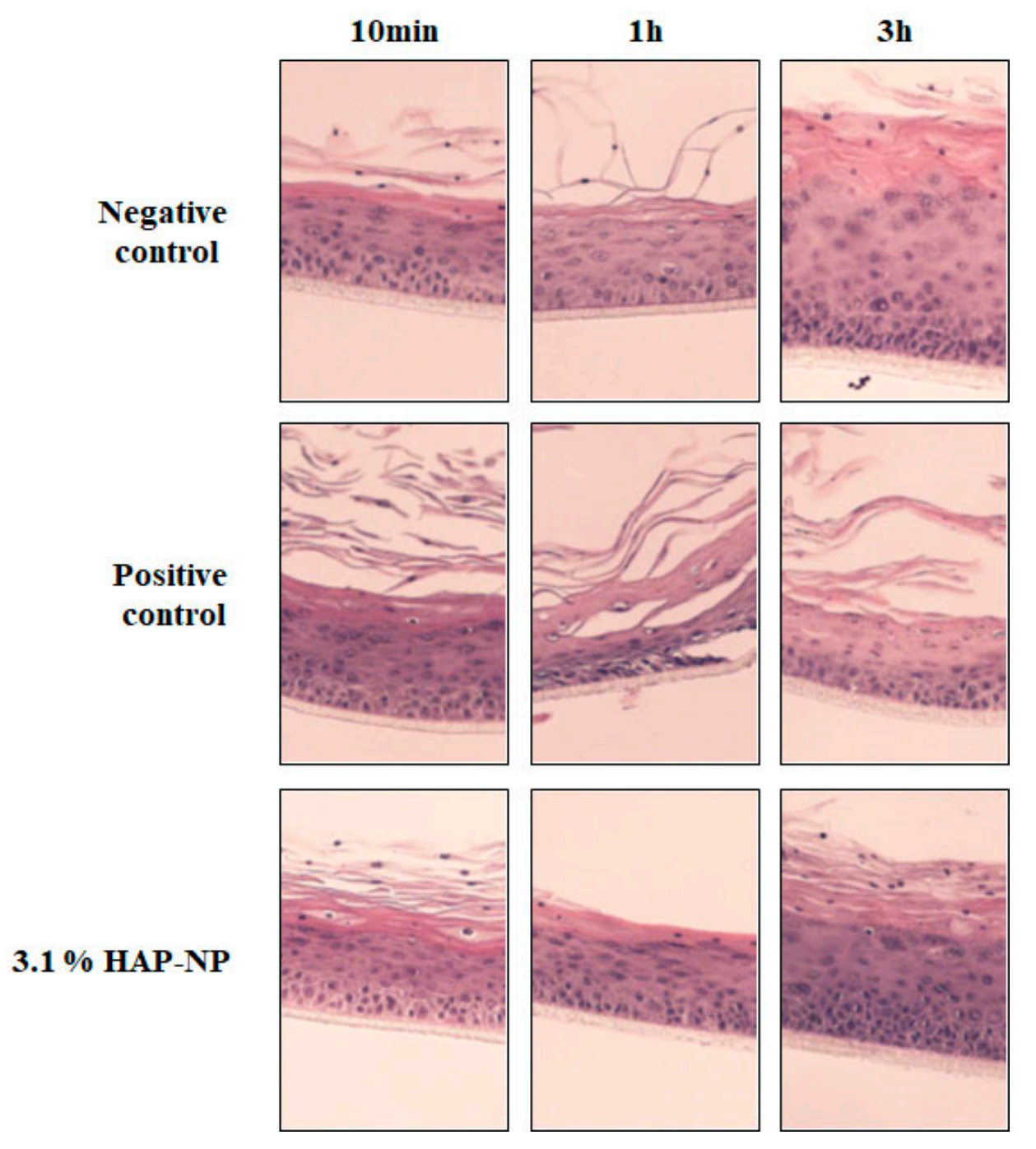

Figure 3. Histology of HGE after different exposure times with the treatments. Negative control was obtained by treating HE with $\mathrm{MQ}$ water: PBS (1:1). Positive control was HE treated with $1 \%$ SDS diluted in PBS (1:1). HGE treated with 3.1\% HAP-NP in PBS. Paraffin slices were stained with hematoxylin/eosin.

Table 2. Results with Histopathological scores of HGE tissues after treatments.

\begin{tabular}{cccc}
\hline \multirow{2}{*}{ Group } & \multicolumn{3}{c}{ Score } \\
\cline { 2 - 4 } & Negative Control & Positive Control & $\mathbf{3 . 1}$ \% HAP-NP \\
\hline $10 \min$ & 0 & 0 & 0 \\
$1 \mathrm{~h}$ & 0 & 1 & 0 \\
$3 \mathrm{~h}$ & 0 & 1 & 0 \\
\hline
\end{tabular}

TEM analysis (Figure 4) verified the absence of HAP-NP nanoparticles internalized into the cells after treatment in any of the cell layers (only the superficial layer is shown in Figure 4). None of the sections analysed contained cells with nanoparticles internalized. Some nanoparticles were observed outside the most superficial cell layer in the HGE samples treated with $3.1 \%$ HAP-NP after $1 \mathrm{~h}$ of exposure, which is more likely to be related with insufficient washing after treatment for this time point, since the same did not happened for a longer exposure time of $3 \mathrm{~h}$. 
$10 \mathrm{~min}$

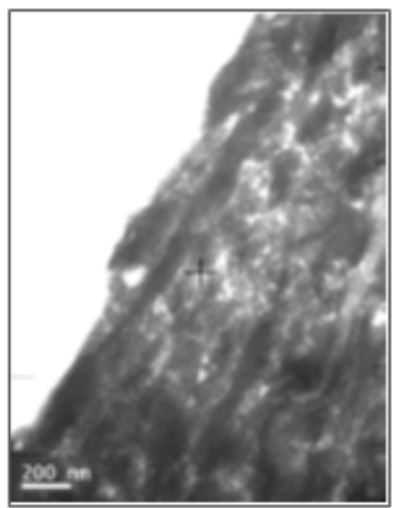

$1 \mathrm{~h}$

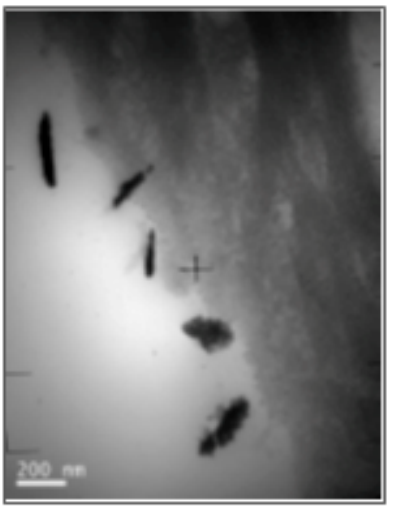

$3 \mathrm{~h}$

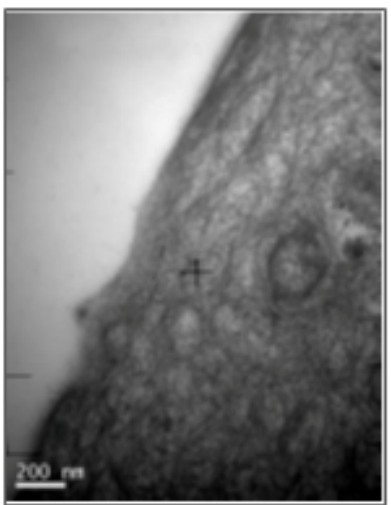

Figure 4. Transmission electron microscopic detection of HAP-NP after 10 minutes, $1 \mathrm{~h}$, and $3 \mathrm{~h}$ incubation of HGE tissues. Images were taken from the most superficial layer at a magnification of $50,000 \times$. A negative and a positive control after $3 \mathrm{~h}$ of incubation were also included in the TEM analyses (data not shown).

\subsection{Calcium Content in the Digestion Media}

Table 3 shows the calcium concentration determined by ICP-AES in the digestion media for $3.1 \%$ HAP-NP after $7.5,15$, and $30 \mathrm{~min}$ at $37^{\circ} \mathrm{C}$. The maximum $\mathrm{Ca}^{2+}$ concentration in the digestion fluid was calculated in order to evaluate the results obtained. Thus, if the total amount of hydroxyapatite nanoparticles were dissolved during the digestion, the concentration of $\mathrm{Ca}^{2+}$ ions in the digestion medium would be of $103 \mathrm{mg} \mathrm{Ca}^{2+} / \mathrm{L}$ for an initial concentration of $3.1 \%$ HAP-NP. Figure 5 shows the amount of $\mathrm{Ca}^{2+}$ released to the media in relation with the maximum concentration expected.

Table 3. Calcium concentration in the digestion media determined by ICP-AES after digestion in simulated gastric fluid (SGF) of an initial concentration of 3.1\% hydroxyapatite nanoparticles (HAP-NP) at different time-points.

\begin{tabular}{cc}
\hline Time (min) & $\mathbf{C a}^{2+}$ Concentration $(\mathbf{m g} / \mathrm{L})$ \\
\hline 7.5 & $104 \pm 3$ \\
15 & $104 \pm 2$ \\
30 & $100 \pm 4$
\end{tabular}

The calculated maximum $\mathrm{Ca}^{2+}$ concentration in the digestion fluid if all the HAP-NP were dissolved would be 103 $\mathrm{mg} \mathrm{Ca}{ }^{2+} / \mathrm{L}$. Values represent mean \pm SEM ( $\mathrm{n}=3$ replicates).

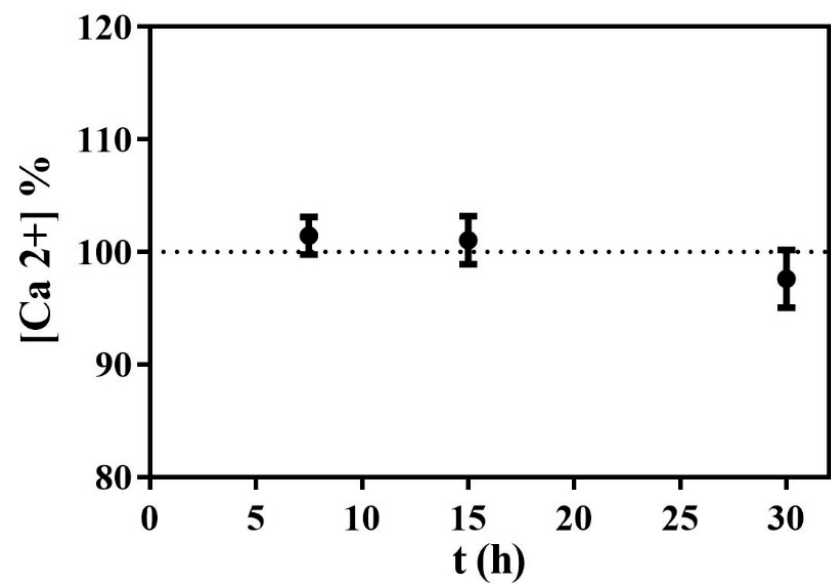

Figure 5. Released calcium ions to the digestion media from 3.1\% HAP-NP suspension in simulated gastric fluid (SGF) at different time points. The calcium concentration is represented in relation to the maximum $\mathrm{Ca}^{2+}$ concentration expected if the totality of the nanoparticles were dissolved. 
As the Table 3 and Figure 5 show, the concentration of $\mathrm{Ca}^{2+}$ in the digestion fluid was similar to the maximum $\mathrm{Ca}^{2+}$ concentration expected at all time-points. Thus, the totality of HAP-NP was dissolved in the SGF at $7.5 \mathrm{~min}$ of incubation at $37^{\circ} \mathrm{C}$. Moreover, it should be highlighted that when diluting the HAP-NP suspension into the SGF at $37^{\circ} \mathrm{C}$, all the solution was immediately clear and no particles could be observed. Additionally, after the centrifugation, there was no sediment observed, which reinforces that 3.1\% HAP-NP was fully dissolved in the SGF for all the time points tested.

\section{Discussion}

The present study evidences the safety of the tested HAP-NP aqueous suspension nanoparticles for oral care products, showing no deleterious effect on HGE tissues and a complete dissolution in simulated gastric fluid. All the tests performed were designed to comply with the European policy of non-animal testing for cosmetics safety assessment. A three-dimensional in vitro replacement alternative that resembles human gingival epithelium was used to demonstrate the safety of these HAP-NP for use in oral care products. Cell viability (MTT), cell toxicity (LDH activity), and the pro-inflammatory response (IL-1alpha) induced by a 3.1\% HAP-NP at different time points were assessed.

Hydroxyapatite nanoparticles have been incorporated in different commercial oral care products $[4,18,19]$ and according to the manufacturer, the maximum incorporation of this $15.5 \%$ HAP-NP aqueous suspension oral care ingredient in a toothpaste is $20 \%$ and in a mouthwash is $10 \%$. Considering this, it was decided to test a final concentration of $20 \%$ dilution of the HAP-NP aqueous suspension, which corresponds to 3.1\% HAP-NP. As regards to the exposure time, taking into account that the recommended as well as applied average time for tooth brushing is $2 \mathrm{~min}[20,21]$, considerable longer exposure times were evaluated, namely $10 \mathrm{~min}, 1 \mathrm{~h}$, and $3 \mathrm{~h}$ to ensure that the HAP-NP would be safe on this application even with much longer contact times than the expected.

Both cell viability and cytotoxicity tests showed that exposure to $3.1 \%$ HAP-NP for up to $3 \mathrm{~h}$ is safe. To the best of our knowledge, this is the first study evaluating the safety of HAP-NP in HGE. A safety assessment using mucosa-like human corneal epithelial (HCE) tissue model-using human corneal keratinocytes - has also demonstrated similar results, with a lack of cytotoxicity and no changes in IL-1 alpha release [22]. Usually, safety studies are performed using 2D cell culture models, such as human buccal epithelial cells [23], 3T3 fibroblasts [22] on osteoblast-like cells [24] using different crystallographic nature of the HAP-NP, which can induce different cell responses, as reviewed by Epple [25].

The lack of effect in cell viability induced by the positive control can be explained by the relatively low SDS concentration used in this assay, evidencing the low sensitivity of HGE tissue particularly for MTT test, as reported by others [10]. This probably happens due to the keratinized external layer of this tissue and/or to the relatively low SDS concentration ( $0.5 \%)$ used in this assay. This concentration was selected as is routinely used in this kind of tests in 3D in vitro models and works well for oral epithelium [10], which lacks the protection to the detergent given by the keratinized external layer. However, although no deleterious effect induced by the positive control could be observed by the MTT test, higher cytotoxicity determined by LDH activity and higher IL-1alpha levels were found. IL-1 cytokines are one of the primary initiators of the dermal pro-inflammatory response and are well-known indicators of acute irritation in reconstructed human epidermis [17]. In fact, IL-1alpha has been proposed by the SCCS [12] as a second endpoint in order to obtain better sensitivity. Thus, after $3 \mathrm{~h}$ of exposure, $0.5 \%$ SDS treatment induced a significantly higher release of IL-1alpha compared to the negative control (10-fold increase) and to the HAP-NP test solution. These results are in agreement with previous observations [26], showing that elevated levels of IL-1alpha could be detected without any detectable decrease in cell viability in response to endoplasmic reticulum stress.

To test the possibility of cellular uptake of the HAP-NP, two different tests were performed. The first test evaluated the presence or absence of HAP-NP in the gingival mucosa by observation with TEM. Our results confirmed that at the concentrations evaluated and during the exposure times tested 
there are no cellular uptake by HGE. The second test was performed in case of accidental swallowing and consequent uptake in other zones of the digestive tract. For that purpose, 3.1\% HAP-NP suspension was added to SGF to evaluate the gastric dissolution following the recommendations of the FDA [16] and the exposure doses referred in the SCCS Notes [12].

According to FDA guidelines, a conservative conclusion is that a product undergoing $85 \%$ dissolution in 15 min under mild dissolution conditions in $0.1 \mathrm{~N} \mathrm{HCl}$ behaves like a solution and generally should not have any bioavailability problems [16]. In this work, it was proved that the $3.1 \%$ HAP-NP suspension was fully dissolved in half of this time (Table 3 and Figure 5). This test was done considering the double of the maximum daily concentration of HAP-NP used in a mouthwash in a single ingestion, which is the worst scenario of exposure and ingestion if compared to a toothpaste [12]. Additionally, it should be highlighted that once the HAP-NP 3.1\% suspension (an opaque suspension) is added to the SGF, the dissolution is almost instantaneous leading to a clear solution without any observation of the opacity caused by the HAP-NP particles suspension. This reinforces the conclusion about the safety of the HAP-NP in the case of ingestion, since it will dissolve instantaneously in its $\mathrm{Ca}^{2+}$ and $\mathrm{PO}_{4}{ }^{2-}$ ions, in agreement with the Epple's statement that rules out any adverse health effect by an oral exposition to calcium phosphate [25]. Therefore, the possibility of HAP-NP absorption by intestinal cells after accidental ingestion was also discarded.

\section{Conclusions}

In this study, the characterization and safety assessment was performed taking into consideration the SCCS opinion on hydroxyapatite nanomaterial. Our results evidence the safety of the HAP-NP aqueous suspension for oral care products with the use of an in vitro replacement alternative for human gingival epithelium and a simulated gastric fluid assay.

Author Contributions: Conceptualization, J.M.R., C.C.C., A.C., P.A.Q., M.M.; Methodology, J.M.R., C.C.C., A.C., P.A.Q., M.M.; Formal Analysis, J.M.R., A.C., M.M.; Investigation, J.M.R., C.C.C., A.C., M.M.; Resources, P.A.Q., M.M.; Writing-Original Draft Preparation, J.M.R., M.M.; Writing-Review \& Editing, J.M.R., C.C.C., P.A.Q., M.M.; Visualization, J.M.R., C.C.C., A.C., M.M.; Supervision, M.M., P.A.Q.; Project Administration, M.M.; Funding Acquisition, P.A.Q.

Funding: This work was funded by FLUIDINOVA, S.A.

Acknowledgments: The authors thank Dr. Ferran Hierro (University of the Balearic Islands) and María Pocoví and Dr. Carles Saus (Hospital Universitari Son Espases) for their technical contribution regarding the analysis of the HGE tissues with TEM. The authors also acknowledge Luís Teixeira and Marta Ferro (University of Aveiro) for the characterization of the HAP-NP with TEM.

Conflicts of Interest: The authors declare no conflict of interest. The funders had a role in the design of the study, interpretation of data, writing of the manuscript and in the decision to publish the results.

\section{References}

1. Zhou, H.; Lee, J. Nanoscale hydroxyapatite particles for bone tissue engineering. Acta Biomater. 2011, 7, 2769-2781. [CrossRef] [PubMed]

2. Huang, S.B.; Gao, S.S.; Yu, H.Y. Effect of nano-hydroxyapatite concentration on remineralization of initial enamel lesion in vitro. Biomed. Mater. 2009, 4, 34104. [CrossRef] [PubMed]

3. Vano, M.; Derchi, G.; Barone, A.; Covani, U. Effectiveness of nano-hydroxyapatite toothpaste in reducing dentin hypersensitivity: A double-blind randomized controlled trial. Quintessence Int. 2014, 45, 703-711. [CrossRef] [PubMed]

4. Vano, M.; Derchi, G.; Barone, A.; Pinna, R.; Usai, P.; Covani, U. Reducing dentine hypersensitivity with nano-hydroxyapatite toothpaste: A double-blind randomized controlled trial. Clin. Oral Investig. 2018, 22, 313-320. [CrossRef] [PubMed]

5. Low, S.B.; Allen, E.P.; Kontogiorgos, E.D. Reduction in dental hypersensitivity with nano-hydroxyapatite, potassium nitrate, sodium monoflurophosphate and antioxidants. Open Dent. J. 2015, 92-97. [CrossRef] [PubMed] 
6. Jena, A.; Kala, S.; Shashirekha, G. Comparing the effectiveness of four desensitizing toothpastes on dentinal tubule occlusion: A scanning electron microscope analysis. J. Conserv. Dent. 2017, 20, 269-272. [CrossRef] [PubMed]

7. Tschoppe, P.; Zandim, D.L.; Martus, P.; Kielbassa, A.M. Enamel and dentine remineralization by nano-hydroxyapatite toothpastes. J. Dent. 2011, 39, 430-437. [CrossRef] [PubMed]

8. European Comission Regulation (EC) No 1223/2009 of the European Parliament and of the Council of 30 November 2009 on cosmetic products. OJ L 2009, 342, 59. Available online: https://ec.europa.eu/ health/sites/health/files/endocrine_disruptors/docs/cosmetic_1223_2009_regulation_en.pdf (accessed on 7 September 2018).

9. SCCS (Scientific Committee on Consumer Safety) Guidance on safety assessment of nanomaterials in cosmetics, 26-27 June 2012. Eur. Comm. Available online: http:/ / ec.europa.eu/health/scientific_committees / consumer_safety /docs /sccs_s_005.pdf (accessed on 7 September 2018).

10. Wurzburger, L.; Kazmi, P.; Re, T.; Alonso, A.; Bertin, B.; Barnes, N.; de Brugerolle de Fraissinette, A.; Hilberer, A.; Raabe, H.; Wilt, N.; et al. Evaluation of an Oral Care Product Safety Screening Program Utilizing the In Vitro SkinEthic Human Gingival Epithelium (RHG) and Oral Buccal (RHO) Models. In Proceedings of the 50th SOT Annual Meeting, Washington, DC, USA, 6-10 March 2011.

11. LLopis-Grimalt, M.; Munar-Bestard, M.; Ramis, J.M.; Monjo, M. Tissue-Engineered Oral Mucosa Constructs for in Vitro Research and Clinical Applications. Biomed. J. Sci. Tech. Res. 2018, 2, 3-5. [CrossRef]

12. SCCS (Scientific Committee on Consumer Safety) The SCCS Notes of Guidance for the Testing of Cosmetic Ingredients and Their Safety E Valuation, 9th revision 2015. Available online: http:/ / ec.europa.eu/health/ scientific_committees/consumer_safety/docs/sccs_o_190.pdf (accessed on 7 September 2018).

13. Pauwels, M.; Rogiers, V. Human health safety evaluation of cosmetics in the EU: A legally imposed challenge to science. Toxicol. Appl. Pharmacol. 2010, 243, 260-274. [CrossRef] [PubMed]

14. SCCS (Scientific Committee on Consumer Safety) Opinion on Hydroxyapatite (nano), 16 October 2015, 2016, revision of 16 March 2016. Available online: https:/ / ec.europa.eu/health/scientific_committees/consumer_ safety/docs/sccs_o_191.pdf (accessed on 7 September 2018).

15. de Brugerolle, A. SkinEthic Laboratories, a company devoted to develop and produce in vitro alternative methods to animal use. ALTEX 2007, 24, 167-171. [CrossRef] [PubMed]

16. Food and Drug Administration Guidance for Industry: Dissolution Testing of Immediate Release Solid Oral Dosage Forms 1997, 4. Available online: https:/ /www.fda.gov/downloads/drugs/guidances/ucm070237. pdf (accessed on 7 September 2018).

17. Casas, J.W.; Lewerenz, G.M.; Rankin, E.A.; Willoughby, J.A.; Blakeman, L.C.; McKim, J.M.; Coleman, K.P. In vitro human skin irritation test for evaluation of medical device extracts. Toxicol. In Vitro 2013, 27, 2175-2183. [CrossRef] [PubMed]

18. Anand, S.; Rejula, F.; Sam, J.V.G.; Christaline, R.; Nair, M.G.; Dinakaran, S. Comparative Evaluation of Effect of Nano-hydroxyapatite and 8\% Arginine Containing Toothpastes in Managing Dentin Hypersensitivity: Double Blind Randomized Clinical Trial. Acta Medica 2017, 60, 114-119. [CrossRef] [PubMed]

19. Vano, M.; Derchi, G.; Barone, A.; Genovesi, A.; Covani, U. Tooth bleaching with hydrogen peroxide and nano-hydroxyapatite: A 9-month follow-up randomized clinical trial. Int. J. Dent. Hyg. 2015, 13, 301-307. [CrossRef] [PubMed]

20. Winterfeld, T.; Schlueter, N.; Harnacke, D.; Illig, J.; Margraf-Stiksrud, J.; Deinzer, R.; Ganss, C. Toothbrushing and flossing behaviour in young adults-A video observation. Clin. Oral Investig. 2015, 19, 851-858. [CrossRef] [PubMed]

21. Creeth, J.E.; Gallagher, A.; Sowinski, J.; Bowman, J.; Barrett, K.; Lowe, S.; Patel, K.; Bosma, M.L. The effect of brushing time and dentifrice on dental plaque removal in vivo. J. Dent. Hyg. 2009, 83, 111-116. [PubMed]

22. Scheel, J.; Hermann, M. Integrated risk assessment of a hydroxyapatite-protein-composite for use in oral care products: A weight-of-evidence case study. Regul. Toxicol. Pharmacol. 2011, 59, 310-323. [CrossRef] [PubMed]

23. Tay, C.Y.; Fang, W.; Setyawati, M.I.; Chia, S.L.; Tan, K.S.; Hong, C.H.L.; Leong, D.T. Nano-hydroxyapatite and nano-titanium dioxide exhibit different subcellular distribution and apoptotic profile in human oral epithelium. ACS Appl. Mater. Interfaces 2014, 6, 6248-6256. [CrossRef] [PubMed]

24. Shi, Z.; Huang, X.; Cai, Y.; Tang, R.; Yang, D. Size effect of hydroxyapatite nanoparticles on proliferation and apoptosis of osteoblast-like cells. Acta Biomater. 2009, 5, 338-345. [CrossRef] [PubMed] 
25. Epple, M. Review of potential health risks associated with nanoscopic calcium phosphate. Acta Biomater. 2018, 77, 1-14. [CrossRef] [PubMed]

26. Suwara, M.I.; Green, N.J.; Borthwick, L.A.; Mann, J.; Mayer-Barber, K.D.; Barron, L.; Corris, P.A.; Farrow, S.N.; Wynn, T.A.; Fisher, A.J.; et al. IL-1 $\alpha$ released from damaged epithelial cells is sufficient and essential to trigger inflammatory responses in human lung fibroblasts. Mucosal Immunol. 2014, 7, 684-693. [CrossRef] [PubMed] 